Bangladesh J. Bot. 42(2): 315-320, 2013 (December)

\title{
NEW METHOD FOR SCREENING RICE VARIETIES AGAINST BAKANAE DISEASE
}

\author{
KS Hossain*, MAT MIA ${ }^{1}$ AND MA BASHAR \\ Department of Botany, University of Dhaka, Dhaka-1000, Bangladesh
}

Key words: Bakanae disease, Mass screening, Rice

\begin{abstract}
Several varietal screening methods were tested along with dipping dry seeds into millipore membrane filtrate of spore suspension of the pathogen and gibberellic acid $\left(\mathrm{GA}_{3}\right)$ in different concentrations. It was found that $\mathrm{GA}_{3}$ can be used to screen out susceptible rice varieties against bakanae disease. The method which requires the use of $\mathrm{GA}_{3}$ is easier than the other methods for mass screening as it does not require maintaining living culture of the pathogen. The protocol of inoculum free varietal screening method for bakanae disease of rice was developed to find out resistant varieties from the huge collections of germplasm bank.
\end{abstract}

\section{Introduction}

Rice (Oryza sativa L.) is the predominant staple food for 34 countries of the world. More than $60 \%$ of the world population depends on rice for their carbohydrate in diet. In Bangladesh, rice occupies about $80 \%$ of the total cropped area and about $92 \%$ of the total food grains produced in the country (Anonymous 2004, 2006).

Like other crops, rice also suffers from many plant diseases that play an important role in determining the amount and cost of food. It has been reported that rice is affected by more than 60 diseases; among these, 31 are reported from Bangladesh, of which ten including bakanae are considered as major. It was found in the field that almost all cultivated rice varieties in Bangladesh are more or less susceptible to bakanae disease and up to $26.7 \%$ yield loss was reported owing to the disease. The disease is widely distributed in all rice growing areas of the world. The causal organism of the disease is Fusarium moniliforme Sheldon (Miah et al. 1985, Ou 1985, Latif et al. 2006, Hossain et al. 2011).

The ultimate goal of all phytopathological studies is to control plant diseases and reduce yield losses. The best way of disease control is to grow resistant varieties because it is the economic and environmentally safe method of 'crop disease management' strategies. Unfortunately, a few reports on resistance against bakanae have been reported to date. From Bangladesh, Haque et al. (1979) and Latif et al. (2006) reported some laboratory methods instead of field method for screening of bakanae resistant rice variety that depends on living inoculum production. Consequently, considering the importance of this disease in Bangladeshi context, the present research project was undertaken to develop a protocol on inoculum free method for screening rice varieties against bakanae disease.

\section{Materials and Methods}

Conidial suspensions (conidia/ml) from Fusarium moniliforme Sheldon having different densities of conidia were used on rice (Oryza sativa L.) BR-26 variety in five different sets of experiment. The conidial suspension, hereinafter, has been termed as spore suspension (SS).

*Author for correspondence. Present address: Department of Botany, Jagannath University, Dhaka, Bangladesh. <ksh1968@gmail.com>. ${ }^{1}$ Ex-Chief Scientific Officer and Head, Plant Pathology Division, BRRI, Gazipur, Bangladesh. 
For the preparation of SS, F. moniliforme was isolated from bakanae infected BR-26. The pure culture was maintained on sucrose nutrient agar (SNA) slants kept in a refrigerator. On the mycelial mat of each 9-day-old culture of the pathogen on potato sucrose agar (PSA) medium in Petri plates (90 mm dia.), $10 \mathrm{ml}$ of sterilized distilled water was poured in. The culture surface was then scrapped gently with a sterile glass slide to make a primary SS which was filtered through three-folds sterilized water saturated gauze to avoid mycelial fragments. The density of conidia in the SS was counted by haemocytometer. Requisite amount of sterilized distilled water was added in the SS to achieve desired concentration of conidia. A total of six concentrations of SS were used. These are, $1=5 \times 10^{10}, 2=1 \times 10^{10}, 3=1 \times 10^{9}, 4=1 \times 10^{8}, 5=1 \times 10^{7}$ and $6=1 \times 10^{6}$ conidia $/ \mathrm{ml}$.

One hundred for each of dry seeds, water soaked ( $24 \mathrm{hrs}$ ) seeds, sprouted seeds, four-day-old seedlings (3.5 - $4.0 \mathrm{~cm}$ tall) and seven-day-old seedlings ( $8.0-8.5 \mathrm{~cm}$ tall) of a known susceptible BR-26 were placed into $8 \mathrm{ml}$ of SS $\left(5 \times 10^{10}\right.$ conidia/ml) in Petri plates (90 mm dia.). Among the treatments, first three were seed inoculation and last two were root deep method. The Petri plates were cultured in vitro at room temperature under fluorescent light for 10 days. From the 3rd day of inoculation requisite amount of sterilized distilled water was added into the plates to prevent desiccation. Everyday room temperature was recorded with a digital thermometer. Thereafter, the percentages of over growth rate (OGR), death rate, lankiness and color of the rice specimens were determined. Effects of different inoculum concentration (SS 1-5), sterilized corresponding suspension filtrate (SCSF), autoclaved spore suspension (ASS) and 9 different concentrations (0.001-1000 ppm) of $\mathrm{GA}_{3}$ on BR-26 were also carried out in the same manner as described above.

SCSF was prepared by centrifuging one set of 1-5 SS for $15 \mathrm{~min}$ at $5000 \mathrm{rpm}$. The supernatant was separated gently and were passed through millipore membrane filter (Sartorius, pore dia. $0.45 \mu \mathrm{m}, 25 \mathrm{~mm}$ circles) to eliminate all germs from the suspensions. A second set of similar supernatant from SCSF was autoclaved at $121^{\circ} \mathrm{C}$ for 15 minutes and marked as ASS. All the experiments regarding SS 1-5, SCSF and ASS were carried out with dry seeds of BR-26. There were three replicates in each set and a control containing the same amount of sterile distilled water instead of SS.

\section{Results and Discussion}

Effects of spore suspension $\left(5.0 \times 10^{10}\right.$ conidia/ml) of $F$. moniliforme on different growth stages of BR-26 were presented in the Table 1. Disease intensity in term of OGR was found the highest $(128.7 \%)$ in dry seed inoculation and then gradually decreased in water soaked seeds (118.6\%), sprouted seeds (104.4\%) and 4-day (95.9\%) and 7-day (91.2\%) old seedlings. Similar pattern was recorded in per cent death over untreated control sets.

Table 1. Effects of spore suspension of Fusarium moniliforme on seeds and seedlings at different growth stages of BR-26 rice variety.

\begin{tabular}{lccccc}
\hline \multirow{2}{*}{ Parameters } & \multicolumn{5}{c}{ Growth stages } \\
\cline { 2 - 6 } & $\begin{array}{c}\text { Dry } \\
\text { seeds }\end{array}$ & $\begin{array}{c}\text { Water soaked } \\
(24 \mathrm{hrs}) \\
\text { seeds }\end{array}$ & $\begin{array}{c}\text { Sprouted } \\
\text { seeds }\end{array}$ & $\begin{array}{c}4 \text { days old } \\
\text { seedlings } \\
(3.5-4.0 \mathrm{~cm})\end{array}$ & $\begin{array}{c}7 \text { days old } \\
\text { seedlings } \\
(8.0-8.5 \mathrm{~cm})\end{array}$ \\
\hline OGR (\%) & 128.7 & 118.6 & 104.4 & 95.9 & 91.2 \\
Death rate (\%) & 28.8 & 15.9 & 5.4 & 3.8 & 3.1 \\
\hline
\end{tabular}

OGR= Overgrowth rate, treatments with all kinds of seeds and seedlings showed lanky and pale green in colour.

In contrast with the present experiment, Haque et al. (1979) reported that maximum disease development both qualitatively and quantitatively was found in dipping just sprouted seeds in 
spore suspension before sowing for $12 \mathrm{hrs}$. The difference with the present study might be owing to the differences in methodology and/or pathogenic strain.

Results of the present investigation could be compared with the findings of Rajagopalan and Bhuvaneswari (1964). They reported that sowing ungerminated seeds in infected soil resulted in rapid progress of the disease and a high percentage of mortality. Whereas, mild disease symptoms resulted when germinated seeds were sown.

Higher OGR and death rate might be the indication of higher susceptibility of a rice variety against bakanae disease that was found in dry seeds inoculation in the present investigation. Therefore, incubation of dry seeds in SS $\left(5.0 \times 10^{10}\right.$ conidia/ml) of a pathogenic strain under fluorescent light for 10 days might be considered a suitable laboratory method to screen out susceptible rice varieties against bakanae disease.

Table 2 shows that pale green and lanky seedlings were found up to $1.0 \times 10^{7}$ conidia $/ \mathrm{ml}$ treated sets. Therefore, minimum effective level of inoculum potential for screening rice varieties against bakanae disease was detected as $1.0 \times 10^{7}$ conidia/ml in this study. Disease intensity was measured in terms of colour, lankiness, OGR and death rate that were found best in the highest inoculum concentration $\left(5.0 \times 10^{10}\right.$ conidia/ml). In artificial inoculation method of varietal screening, higher inoculum concentration is necessary to ensure heavy disease pressure (Haque et al. 1979).

Table 2. Effects of inoculum concentrations of Fusarium moniliforme on rice dry seeds.

\begin{tabular}{|c|c|c|c|c|c|c|c|}
\hline \multirow{2}{*}{ Parameters } & \multicolumn{6}{|c|}{ Inoculum concentrations of the suspension (conidia/ml) } & \multirow{2}{*}{ Control } \\
\hline & $5.0 \times 10^{10}$ & $1.0 \times 10^{10}$ & $1.0 \times 10^{9}$ & $1.0 \times 10^{8}$ & $1.0 \times 10^{7}$ & $1.0 \times 10^{6}$ & \\
\hline Colour & PG & PG & PG & PG & PG & G & G \\
\hline Lankiness & $\mathrm{L}$ & $\mathrm{L}$ & $\mathrm{L}$ & $\mathrm{L}$ & $\mathrm{L}$ & ML & NL \\
\hline OGR (\%) & 145.6 & 140.0 & 130.0 & 104.4 & 68.9 & 35.6 & N/A \\
\hline Death (\%) & 28.0 & 25.3 & 20.0 & 20.0 & 19.2 & 12.7 & 5.3 \\
\hline
\end{tabular}

OGR = Overgrowth rate, $\mathrm{PG}=$ Pale green, $\mathrm{G}=$ Green $\mathrm{L}=$ Lanky, $\mathrm{ML}=$ Moderately lanky, NL = Normal, N/A = Not applicable.

Table 2 also shows that disease intensity decreased with the decrease in inoculum concentration. It is well known that every spore of a pathogen has potentiality to produce disease. Disease intensity cannot differ greatly with the decrease of inoculum potential on such a small population. There might be present something else in the spore suspension responsible for decreasing disease intensity when the concentration of the suspension was decreased. It is also well known that $F$. moniliforme produces growth promoters named gibberellins (GAs). Presence of GAs in the suspension may have an important role in overgrowth of the seedlings. When spore suspension was diluted into different levels, GAs of the suspensions also diluted. As a result, OGR, lankiness and colour were decreased with the decrease of inoculum concentration in this experiment. Therefore, sterilized suspension filtrate of $F$. moniliforme might mimic bakanae symptom.

The results of effects of different concentration of SS, SCSF and ASS of F. moniliforme on dry rice seeds are presented in Fig. 1 which shows that OGR and death rate were higher in SS than SCSF in all inoculum concentrations. This higher OGR and death rates might be due to the presence of pathogenic spores in the suspensions (SS). Fig. 1 also shows that both lines were approximately parallel to each other. That is, OGR of the seedlings in SS and SCSF of $F$. moniliforme are almost perfectly correlated to each other across all inoculum potentials. The overgrowth curve has a positive, decreasing slope at higher inoculum concentrations $\left(5.0 \times 10^{10}-\right.$ 
$1.0 \times 10^{10}$ conidia/ml). This decrease in slope means that after a certain level of inoculum concentration the acceleration of OGR decreases. Hormones in higher concentrations showed similar pattern. Almost the same pattern of effect was observed in ASS (Fig.1). Therefore, the growth promoting factor present in the spore suspension was heat resistant. It should be mentioned that GAs also shows similar effect which might replace spore suspension in testing the susceptibility of rice varieties against bakanae disease. In China, Ma et al. (2008) obtained a significant correlation between the length of the seedling treated with $\mathrm{GA}_{3}$ and disease injury by bakanae fungus.
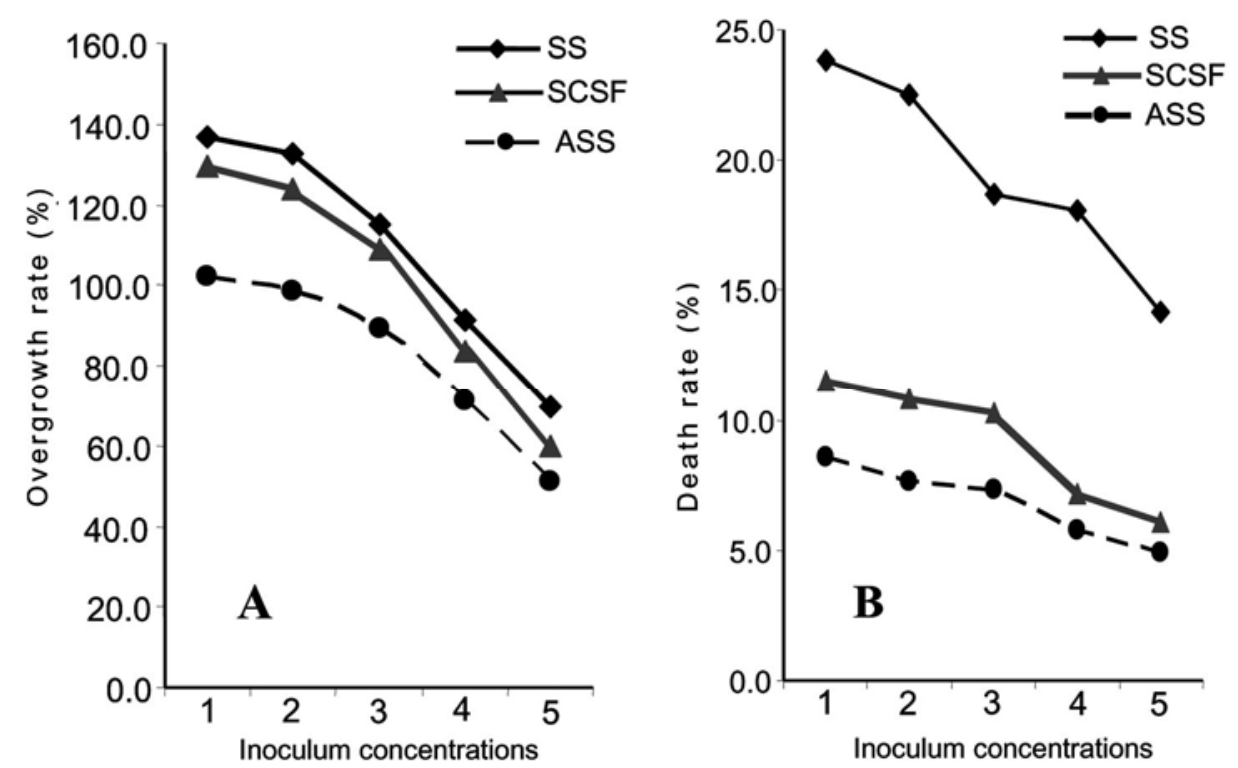

Fig. 1. Comparison of effects of spore suspension (SS), sterilized corresponding suspension filtrate (SCSF) and autoclaved spore suspension (ASS) on dry rice seeds. A. Overgrowth curve and B. Death curve. $1=$ $5 \times 10^{10}, 2=1 \times 10^{10}, 3=1 \times 10^{9}, 4=1 \times 10^{8}, 5=1 \times 10^{7}$.

Effects of SSSF $\left(5.0 \times 10^{10}\right.$ conidia/ml) of $F$. moniliforme and different concentrations of $\mathrm{GA}_{3}$ on dry rice seeds were presented in Table 3 . In comparison with control sets, effects of $\mathrm{GA}_{3}$, on OGR, colour and lankiness, were clearly found at 1 to $1000 \mathrm{ppm}$ concentrations. GA $\mathrm{G}_{3}$ of 0.01 and $0.1 \mathrm{ppm}$ and $0.001 \mathrm{ppm}$ had less and no effects on the growth of the rice seedlings respectively.

Table 3. Effects of different concentrations (ppm) of $\mathrm{GA}_{3}$ and sterilized spore suspension filtrate (SSSF) of Fusarium moniliforme on dry rice seeds.

\begin{tabular}{lccccccccccc}
\hline \multirow{2}{*}{ Parameters } & \multirow{2}{*}{ Control } & \multicolumn{8}{c}{ Different concentrations (ppm) of GA $\mathrm{GA}_{3}$} & SSSF \\
\cline { 3 - 11 } & & 0.001 & 0.01 & 0.1 & 1.0 & 10.0 & 50.0 & 100 & 500 & 1000 & $\left(5.0 \times 10^{10}\right)$ \\
\hline OGR (\%) & N/A & 0.0 & 3.2 & 7.9 & 38.1 & 77.8 & 87.3 & 125.4 & 134.5 & 131.7 & 130.2 \\
Colour & G & G & G & G & PG & PG & PG & PG & PG & PG & PG \\
Lankiness & NL & NL & NL & NL & L & L & L & L & L & L & L \\
\hline
\end{tabular}

Abbreviations are same as Table 2.

Effects of the SSSF were comparable to that found at $100 \mathrm{ppm}$ of $\mathrm{GA}_{3}$. Considerable decrease of OGR was noted at $1000 \mathrm{ppm} \mathrm{GA}_{3}$. Therefore, instead of $5.0 \times 10^{10}$ conidia/ml spore suspension, 
$100 \mathrm{ppm} \mathrm{GA}_{3}$ might be suitable for the test of rice varieties against bakanae disease. Effects of $\mathrm{GA}_{3}$ have to be ensured clearly by using a control set side by side.

The effect of temperature $\left({ }^{\circ} \mathrm{C}\right)$ on rice seedlings height $(\mathrm{cm})$ untreated and treated in vitro with SS and 100 ppm GA 3 presented in the Fig. 2. The figure shows that seedling heights of untreated control sets increased with the increase of temperature but, acceleration of the average heights with the increase of temperature in SS and $\mathrm{GA}_{3}$ treated sets were much higher than that of the control set. Therefore, temperature had an influence on OGR of diseased plants. The Fig. 2 also shows that acceleration in SS treated sets was slightly higher than that of $\mathrm{GA}_{3}$ treated sets. It is well known that pathogen produce gibberellins including $\mathrm{GA}_{3}$ and this might be owing to the combined action of gibberellins produced by the pathogen present in the suspension.

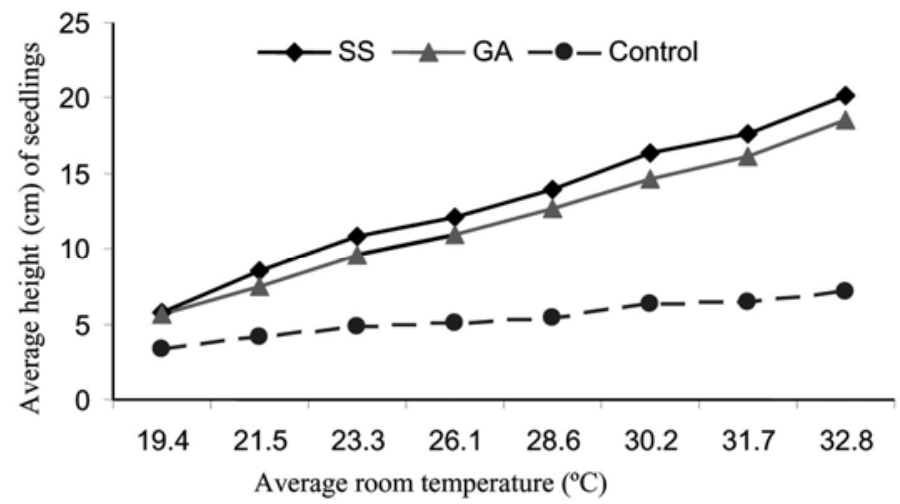

Fig. 2. Effects of temperature $\left({ }^{\circ} \mathrm{C}\right)$ on average height $(\mathrm{cm})$ of treated seedlings. $\mathrm{SS}=$ Spore suspension, $\mathrm{GA}=$ $\mathrm{GA}_{3}$, and Control $=$ non-treated seedlings.

Seto $(1933,1935)$ found that a temperature of $35^{\circ} \mathrm{C}$ is most favourable for seed growth and also for seed infection. The favourable temperature for the growth of the fungus was about 27-30 ${ }^{\circ} \mathrm{C}$. In the present study, in $\mathrm{GA}_{3}$ treated seeds (Table 4), maximum OGR (158.3\%) was found in the maximum temperature range $\left(29.1-36.0^{\circ} \mathrm{C}\right)$.

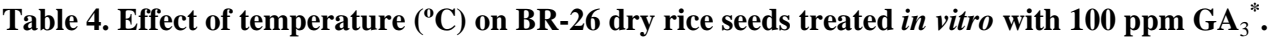

\begin{tabular}{ccccccccc}
\hline & \multicolumn{6}{c}{ Minimum-maximum room temperature $\left({ }^{\circ} \mathrm{C}\right)$ during 10 days of each set of inoculation } \\
\cline { 2 - 8 } & $15.1-20.0$ & $17.5-25.8$ & $18.8-27.7$ & $22.6-30.5$ & $25.3-32.3$ & $27.8-32.6$ & $29.2-34.4$ & $29.1-36.0$ \\
\hline OGR (\%) & 69.70 & 78.60 & 100.00 & 115.70 & 135.20 & 131.70 & 151.60 & 158.30 \\
\hline
\end{tabular}

OGR $=$ Over growth rate

Therefore, a brief protocol for an inoculum free method for screening rice varieties against bakanae disease may be suggested as follows: (i) Dry seeds have to be inoculated into $100 \mathrm{ppm}$ aqueous solution of $\mathrm{GA}_{3}$., (ii) subsequently these have to be incubated at $30-35^{\circ} \mathrm{C}$ for 10 days under florescent tube light on the laboratory table. and (iii) a known susceptible variety should be included simultaneously.

In 2008, it was reported from China by Ma et al. that rice materials carrying dwarf gene such as $s d 1$ were not only sensitive to $\mathrm{GA}_{3}$ but also susceptible to rice bakanae disease. Materials carrying dwarf genes $d 29$, $s d 6$ or $s d q(\mathrm{t})$ were insensitive to $\mathrm{GA}_{3}$ and resistant to bakanae. The present study indicated that $\mathrm{GA}_{3}$ insensitive dwarf and semi-dwarf rice materials might be useful resources for improvement of bakanae resistance in rice breeding programs. The method describe 
herein is a quick one and would not require the maintenance of living culture plates of the pathogen.

\section{Acknowledgements}

The first author gratefully acknowledges to the National University, Gazipur, Bangladesh for providing financial assistance to this research project in the form of a research fellowship. The authors are also thankful to Bangladesh Rice Research Institute (BRRI), Gazipur, for providing laboratory and field facilities during the course of this work.

\section{References}

Anonymous 2004. Rice and human nutrition-fact sheet 3, International year of rice 2004. Food and Agricultural Organization (FAO), Rome, Italy.

Anonymous 2006. FAO Rice Market Monitor. Food and Agricultural Organization (FAO), Rome, Italy. Vol. IX (March), Issue No. 1.

Haque MA, Rahman MM and Miah SA 1979. A rapid method of screening rice varieties for resistance to bakanae disease. IRC Newslet. 28(2):34-37.

Hossain KS, Mia MAT and Bashar MA 2011. Preferred rice varieties, seed source, disease incidence and loss assessment to bakanae disease. J. Agrofor. Environ. 5(2):125-128.

Latif MA, Mia MAT and Hossain MA 2006. Yield loss, integrated management approaches and development of quick screening method of bakanae disease of rice in Bangladesh. Abstract, 2nd international rice congress, October 9-13, 2006. New Delhi, India.

Ma L, Ji Z, Bao J, Zhu X, Li X, Zhuang J, Yang C and Xia Y 2008. Responses of rice genotypes carrying different dwarf genes to Fusarium moniliforme and gibberellic acid. Pl. Produc. Sci. 11(1):134-138.

Miah S A, Shajahan AKM, Hossain MA and Sharma NR 1985. A survey of rice diseases in Bangladesh. Trop. Pest Manag. 31(3): 208-213.

Ou SH 1985. Rice Disease. 2nd ed. CAB International Mycological Institute, Kew, Surrey, U.K. pp. XI + 380.

Rajagopalan K and Bhuvaneswari K 1964. Effect of germination of seeds and host exudations during germination on foot rot disease of rice. Phytopathol. Zeitschrift 50: 221-226. (Quoted by Ou 1985).

Seto F 1933. Untersuchungen über die 'Bakanae'- krankheit der Reispflanze. III. Über die Beziehungen Zwischen der Bodenfeuchtigkeit und dem Krankheitsbefall durch Biodeninfektion. Forschungen aus dem Gebiet der Pflanzenkrankheiten. 2: 125-137. (Quoted by Ou 1985).

Seto F 1935. Beitrage zur Kenntnis der 'Bakanae'- krankheit der Reispflanze. Memoirs of the College of Agriculture, Kyoto Univ., 36:1-81. (Quoted by Ou 1985). 